\title{
A construction of chiral fermion action
}

\author{
Ting-Wai Chiu ${ }^{1}$ \\ Department of Physics, National Taiwan University, Taipei, 106 Taiwan, ROC
}

Received 7 September 1999; received in revised form 1 October 1999; accepted 4 October 1999

Editor: M. Cvetič

\begin{abstract}
According to the necessary requirements for a chirally symmetric Dirac operator, we present a systematic construction of such operators. We formulate a criterion for the hermitian operator which enters the construction such that the doubled modes are decoupled even at finite lattice spacing. (C) 1999 Published by Elsevier Science B.V. All rights reserved.
\end{abstract}

PACS: 11.15.Ha; 11.30.Rd; 11.30.Fs

Recently we have discussed the necessary requirements [1] for a chirally symmetric Dirac operator $D_{c}$ to enter the topologically invariant transformation $D=D_{c}\left(\mathbb{1}+R D_{c}\right)^{-1}[2]$ such that the resulting Dirac operator $D^{2}$ could reproduce the continuum physics, where $R$ is a hermitian operator which is local in the position space and trivial in the Dirac space. These constraints for $D_{c}$ are:

(a) $D_{c}$ agrees with $\gamma_{\mu}\left(\partial_{\mu}+i A_{\mu}\right)$ in the classical continuum limit.

(b) $D_{c}$ is nonlocal.

(c) $D_{c}$ is free of species doubling.

(d) $D_{c}$ is well defined in topologically trivial background gauge field.

(e) $D_{c}$ has zero modes as well as simple poles ( or equivalently, $V$ has real eigenvalue pairs \pm 1 ) in topologically non-trivial background gauge

\footnotetext{
${ }^{1}$ E-mail: twchiu@phys.ntu.edu.tw

${ }^{2}$ Note that $D$ satisfies the Ginsparg-Wilson relation [3] automatically.
}

fields. Furthermore, the zero modes of $D_{c}$ satisfy the Atiyah-Singer index theorem for any prescribed smooth gauge background.

In this paper, we attempt to construct such chirally symmetric Dirac operator $D_{c}$ which also satisfies the hermiticity condition ${ }^{3}$

$\gamma_{5} D_{c} \gamma_{5}=D_{c}^{\dagger}$.

The hermiticity condition and the chiral symmetry of $D_{c}$ implies that $D_{c}$ is antihermitian, thus there exists one to one correspondence between $D_{c}$ and a unitary operator $V$,

$D_{c}=(\mathbb{I}+V)(\mathbb{1}-V)^{-1}$,
$V=\left(D_{c}-\mathbb{1}\right)\left(D_{c}+\mathbb{1}\right)^{-1}$.

where $V$ also satisfies the hermiticity condition

\footnotetext{
${ }^{3}$ This implies that $D$ also satisfies the hermiticity condition $\gamma_{5} D \gamma_{5}=D^{\dagger}$, and $\operatorname{det}(D)$ is real and positive.
} 
$\gamma_{5} V \gamma_{5}=V^{\dagger}$. Then the unitary operator $V$ can be expressed in terms of a hermitian operator $h$,

$$
\begin{aligned}
V & =\gamma_{5} h=\left(\begin{array}{cc}
\mathbb{1} & 0 \\
0 & -\mathbb{1}
\end{array}\right)\left(\begin{array}{ll}
h_{1} & h_{2} \\
h_{2}^{\dagger} & h_{3}
\end{array}\right) \\
& =\left(\begin{array}{cc}
h_{1} & h_{2} \\
-h_{2}^{\dagger} & -h_{3}
\end{array}\right)
\end{aligned}
$$

where $h_{1}^{\dagger}=h_{1}$ and $h_{3}^{\dagger}=h_{3}$. Using the unitarity condition $V^{\dagger} V=\mathbb{1}$, we have $h^{2}=\mathbb{1}$,

$h^{2}=\left(\begin{array}{cc}h_{1}^{2}+h_{2} h_{2}^{\dagger} & h_{1} h_{2}+h_{2} h_{3} \\ h_{2}^{\dagger} h_{1}+h_{3} h_{2}^{\dagger} & h_{2}^{\dagger} h_{2}+h_{3}^{2}\end{array}\right)=\left(\begin{array}{ll}\mathbb{1} & 0 \\ 0 & \mathbb{1}\end{array}\right)$

Then we obtain [4]

$$
\begin{aligned}
D_{c} & \equiv\left[\begin{array}{cc}
0 & D_{R} \\
D_{L} & 0
\end{array}\right] \\
& =\left[\begin{array}{cc}
0 & \left(\mathbb{1}-h_{1}\right)^{-1} h_{2} \\
-h_{2}^{-1}\left(\mathbb{1}+h_{1}\right) & 0
\end{array}\right]
\end{aligned}
$$

where $D_{L}=-D_{R}^{\dagger}$. The general solution to Eq. (4) can be written as

$$
\begin{aligned}
& h_{1}= \pm \frac{1}{\sqrt{\mathbb{1}+b b^{\dagger}}} \\
& h_{2}=\frac{1}{\sqrt{\mathbb{1}+b b^{\dagger}}} b \mathrm{e}^{i \theta}
\end{aligned}
$$

where $\mathrm{e}^{i \theta}$ is an arbitrary phase, and $b$ is any operator. In the following we will restrict $\theta$ to zero, and also pick the minus sign for $h_{1}$. Then the general solution for $D_{c}$ can be written in the following form

$D_{L}=b^{-1}\left[\mathbb{1}-\sqrt{\mathbb{1}+b b^{\dagger}}\right]$

$D_{R}=\left[\mathbb{1}+\sqrt{\mathbb{1}+b b^{\dagger}}\right]^{-1} b$

Due to the presence of the square root in (8) and (9), $D_{c}$ is nonlocal for nontrivial $b$, thus the requirement (b) is satisfied. Then the Nielson-Ninomiya no-go theorem [5] is circumvented. Hence, we can construct $D_{c}$ to be free of species doubling. If $D_{c}$ is topologically proper [ i.e., satisfying the constraint (e) ], then we can use the topologically invariant transformation $D=D_{c}\left(\mathbb{I}+R D_{c}\right)^{-1}$ to obtain a local $D$ which reproduces correct chiral anomaly on a finite lattice for smooth background gauge fields. Therefore, we must explicitly check whether the constraint (e) is satisfied or not, while the constraint (d) is usually fulfilled except for bizarre cases.

In order to have $D_{c}$ satisfy the constraint (a), we first try

$b=w^{-1} \sum_{\mu} \sigma_{\mu} t_{\mu}$

where

$t_{\mu}(x, y)=\frac{1}{2}\left[U_{\mu}(x) \delta_{x+\hat{\mu}, y}-U_{\mu}^{\dagger}(y) \delta_{x-\hat{\mu}, y}\right]$,

$\sigma_{\mu} \sigma_{\nu}^{\dagger}+\sigma_{\nu} \sigma_{\mu}^{\dagger}=2 \delta_{\mu \nu}$

$\gamma_{\mu}=\left(\begin{array}{cc}0 & \sigma_{\mu} \\ \sigma_{\mu}^{\dagger} & 0\end{array}\right)$

and $w$ is a non-singular hermitian operator which is trivial in the Dirac space and goes to a constant in the classical continuum limit. Then Eqs. (8) and (9) become

$$
\begin{aligned}
& D_{L}=-(\sigma \cdot t)^{-1}\left[\sqrt{w^{2}+w t^{2} w^{-1}}-w\right] \\
& D_{R}=\left[\sqrt{w^{2}+w t^{2} w^{-1}}+w\right]^{-1}(\sigma \cdot t)
\end{aligned}
$$

where

$$
\begin{aligned}
& \sigma \cdot t=\sum_{\mu} \sigma_{\mu} t_{\mu} \\
& t^{2}=-(\sigma \cdot t)\left(\sigma^{\dagger} \cdot t\right)
\end{aligned}
$$

However, $t_{\mu}$ suffers from species doublings. Now our task is to construct a hermitian operator $w$ such that the doubled modes are completely decoupled from $D_{c}$ or the fermion propagator $D_{c}^{-1}$, even at finite lattice spacing. The fermion propagator $D_{c}^{-1}$ is

$$
\begin{aligned}
D_{c}^{-1} & \equiv\left[\begin{array}{cc}
0 & D_{L}^{-1} \\
D_{R}^{-1} & 0
\end{array}\right] \\
& =\left[\begin{array}{cc}
0 & -\left(\mathbb{I}+h_{1}\right)^{-1} h_{2} \\
h_{2}^{-1}\left(\mathbb{1}-h_{1}\right) & 0
\end{array}\right]
\end{aligned}
$$


The left-handed free fermion propagator, $D_{L}^{-1}$, in the momentum space is

$D_{L}^{-1}=-\left[\sqrt{w^{2}+t^{2}}+w\right] \frac{\sigma_{\mu} t_{\mu}}{t^{2}}$

where $t_{\mu}=i \sin \left(p_{\mu} a\right)$ and $t^{2}=\sum_{\mu} \sin ^{2}\left(p_{\mu} a\right)$. The second factor in (19) is exactly the naive fermion propagator which suffers from species doubling at the $2^{d}-1$ corners of the Brillouin zone (BZ), i.e., $\otimes_{\mu}\{0, \pi / a\} \backslash\{p=0\}$. Now we want to construct a hermitian operator $w$ such that $w>0$ for the primary mode at $p=0$ but $w<0$ at the corners of the $\mathrm{BZ}$, then all doubled modes are decoupled completely due to the vanishing of the first factor in (19). Explicitly, we want to construct a hermitian $w$ such that in the free fermion limit, it satisfies the following condition,

$w(p)$

$$
= \begin{cases}>0 & \text { for the primary mode at } p=0, \\ <0 & \text { for the doubled modes at } p \in \otimes_{\mu}\{0, \pi / a\} \backslash\{p=0\} .\end{cases}
$$

It is obvious that $w(p)$ cannot be a simple and smooth function of $\sin \left(p_{\mu} a\right)$ since it vanishes at all corners of the $\mathrm{BZ}$ as well as at the origin $p=0$. The next simplest possibility is $\sin \left(p_{\mu} a / 2\right)$ which vanishes at the origin $p=0$ but not at the corners of BZ. Therefore the candidate

$w(p)=c-2 \sum_{\mu} \sin \left(p_{\mu} a / 2\right), \quad c \in(0,2)$

would satisfy the condition (20). But (21) can only be realized by the symmetric difference operator on a lattice with lattice spacing $a / 2$. If we wish to retain the simple lattice with lattice spacing $a$, we need to modify the form of $\sin \left(p_{\mu} a / 2\right)$ in (21). The simplest modification one can perform is to take the square of $\sin \left(p_{\mu} a / 2\right)$ since $\sin ^{2}\left(p_{\mu} a / 2\right)=[1-$ $\left.\cos \left(p_{\mu} a\right)\right] / 2$ which can be implemented by nearest neighbor difference operators on the lattice with lattice spacing $a$. So, we modify (21) to

$w(p)=c-2 \sum_{\mu} \sin ^{2}\left(p_{\mu} a / 2\right), \quad c \in(0,2)$

It should be emphasized that the role of $w$ in our general solution of $D_{c}$ is quite different from the Wilson term in the Wilson-Dirac operator [6]. In our general solution of $D_{c}$, the chiral symmetry is always preserved, and the role of $w$ is to suppress the doubled modes completely at finite lattice spacing, while in the Wilson-Dirac operator, the Wilson term breaks the chiral symmetry explicitly and gives a mass of order $a^{-1}$ to the doubled modes such that they can be decoupled in the continuum limit ( $a \rightarrow 0)$. After the gauge links are restored, $w$ in the position space becomes

$$
\begin{aligned}
w(x, y)= & c \delta_{x, y}-\frac{1}{2} \sum_{\mu}\left[2 \delta_{x, y}-U_{\mu}(x) \delta_{x+\hat{\mu}, y}\right. \\
& \left.-U_{\mu}^{\dagger}(y) \delta_{x-\hat{\mu}, y}\right]
\end{aligned}
$$

This is one of the simplest solution of $w$ satisfying the requirement (20) in the free field limit. It is plausible that there exists other solutions to (20). Here we do not intend to discuss all possible solutions of $w$, but only to formulate the necessary condition (20) for $w$ to decouple all doubled modes at finite lattice spacing.

Since the $D_{c}$ [ Eqs. (14) and (15) with $t_{\mu}$ and $w$ defined in (11) and (23) ] in the free fermion limit is free of species doubling, and in the momentum space behaves like $i \gamma_{\mu} p_{\mu}$ as $p \rightarrow 0$, the perturbation calculation in Ref. [7] showed that $D=D_{c}\left(\mathbb{I}+r D_{c}\right)^{-1}$ gives the correct chiral anomaly. However, the topological characteristics [1] of a lattice Dirac operator is an intrinsically non-perturbative quantity which in general cannot be revealed by perturbation calculations. Therefore further non-perturbative ( e.g., numerical ) studies are needed before one can understand the topological characteristics of this $D_{c}$. The main objective of this paper is to cast the general solution of $D_{c}$ in the form of (8-9), and to demonstrate a viable construction of the operator $b$.

For any $D_{c}$ satisfying criteria (a)-(e), we can use the topologically invariant transformation $D=D_{c}$ ( $\mathbb{1}$ $\left.+R D_{c}\right)^{-1}$ to obtain a class of local and well defined Dirac fermion operators which not only preserve the physics of $D_{c}$ but also restrict the quantum corrections to behave properly. The fermion propagator and chiral condensate of $D$ have been investigated in Ref. [4].

In summary, we have constructed a class of general solution of the chirally symmetric $D_{c}$ in terms of the hermitian operator $w$ which fulfills the requirement (20) in the free field limit. An example of $w$ is given in (23). One of the salient features of the present formulation is that the chiral fermion opera- 
tor $D_{L}\left(D_{R}\right)$ is obtained explicitly. This may provide a starting point for a formulation of chiral gauge theories on a finite lattice.

\section{Acknowledgements}

This work was supported by the National Science Council, R.O.C. under the grant number NSC892112-M002-017.
[1] T.W. Chiu, Phys. Lett. B445 (1999) 371.

[2] T.W. Chiu, S.V. Zenkin, Phys. Rev. D59 (1999) 074501.

[3] P. Ginsparg, K. Wilson, Phys. Rev. D25 (1982) 2649.

[4] T.W. Chiu, Phys. Rev. D60 (1999) 034503.

[5] H.B. Nielsen, N. Ninomiya, Nucl. Phys. B 185 (1981) 20 [E: B195 (1982) 541]; B193 (1981) 173.

[6] K. Wilson, in: A. Zichichi (Ed.), New Phenomena in Subnuclear Physics, Plenum Press, New York, 1977.

[7] T.W. Chiu, T.H. Hsieh, Perturbation calculation of the axial anomaly of Ginsparg-Wilson fermion, hep-lat/9901011. 